\title{
GRUPOS ESTRATÉGICOS EN EL SECTOR PRIVADO DE LA EDUCACIÓN SUPERIOR
}

(STRATEGIC GROUPS IN PRIVATE HIGHER EDUCATION)

Dorys Yaneth Rodriguez Castro

Jon Mikel Zabala-Iturriagagoitia

Universidad de Deusto

Juan Aparicio

Universidad Miguel Hernández de Elche

DOI: 10.5944/educXX1.26657

Cómo referenciar este artículo/How to reference this article:

Rodríguez Castro, D.Y.; Zabala-Iturriagagoitia, J.M. y Aparicio, J. (2021). Grupos estratégicos en el sector privado de la educación superior. Educación XX1, 24(1), 163-187, http://doi. org/10.5944/educXX1.26657

Rodríguez Castro, D.Y.; Zabala-Iturriagagoitia, J.M. \& Aparicio, J. (2021). Strategic groups in private higher education. Educación XX1, 24(1), 163-187, http://doi.org/10.5944/educXX1.26657

\section{RESUMEN}

El objetivo de este artículo es identificar grupos estratégicos en el sector privado de un sistema de educación superior. Las técnicas de clasificación tienden a comparar conjuntamente a las instituciones de educación superior privadas con sus contrapartes públicas, y corren el riesgo de subestimar el impacto del mercado como fuente de diferenciación institucional. En este artículo, se propone una clasificación que permite superar estas limitaciones. Para ello, se aplican técnicas de clasificación basadas en indicadores de la oferta de formación como variables explicativas de las estrategias de absorción de la demanda. Como resultado, el artículo ofrece una clasificación de las instituciones privadas en un conjunto de grupos estratégicos, y analiza la relación entre sus características institucionales y las estrategias que las diferencian. La aplicación empírica se ha llevado a 
cabo en el sistema de educación superior colombiano, ya que cuenta con uno de los mayores índices de privatización en el mundo. Nuestros resultados indican que la educación a distancia, la oferta de programas técnicos y tecnológicos, y la formación posgradual, son estrategias determinantes en la diferenciación de las instituciones colombianas. La aproximación empleada en el artículo podría utilizarse para abordar las posibles vías de crecimiento o expansión de las instituciones privadas en función de su identidad estratégica, contribuyendo así a la sostenibilidad y competitividad de los sistemas de educación superior.

\section{PALABRAS CLAVE}

Grupos estratégicos, Sector privado, Educación Superior, clasificaciones universitarias

\section{ABSTRACT}

The goal of this paper is to characterize the institutional diversity of the private side of a higher education system. The classification techniques used in the literature have a propensity to compare private higher education institutions with their public counterparts and run the risk of underestimating the impact of the market as a source of institutional differentiation. In this paper we propose an alternative classification that allows this limitation to be overcome. We obtain a multidimensional classification of private higher education institutions and analyze the relationship between their institutional characteristics and the sources of differentiation. The study has been conducted in the Colombian higher education system, as it constitutes one of the systems with the highest rates of privatization worldwide. Our results identify five strategic groups of private higher education institutions. Distance learning, the offer of technical and technological programs, and postgraduate training are key factors determining the differences across Colombian private higher education institutions. The approach followed in this paper could be used to identify potential paths for the development and expansion of private higher education institutions, further contributing to the sustainability and competitiveness of higher education systems.

\section{KEYWORDS}

Strategic groups, Private sector, Higher Education, University classification 
DORYS YANETH RODRIGUEZ CASTRO, JON MIKEL ZABALA-ITURRIAGAGOITIA, JUAN APARICIO

\section{INTRODUCCIÓN}

Los análisis de diversidad se han aplicado en muchos campos de la educación superior (Huisman et al., 2015) Sin embargo, a pesar de su relevancia en el crecimiento y sostenibilidad de los sistemas de educación superior (Kwiek, 2018), son pocos los ejemplos de su aplicación en el sector privado (Álvarez, 2013; Levy, 2009). La literatura se ha centrado principalmente en el diseño de tipologías que clasifican a las Instituciones de Educación Superior privadas (IESp) junto con sus contrapartes públicas, considerando que tanto las instituciones privadas como las públicas son homogéneas entre sí (p.e., Navas et al., 2020), lo que dista mucho de la realidad. Las IESp, por su propia naturaleza, deben implementar estrategias que les permitan competir por los mejores recursos, estudiantes y profesores (Brunner, 2009). Y cuando los recursos son escasos, las instituciones privadas deben desarrollar una oferta de formación que les permita llegar a otros nichos de mercado y atraer nuevos usuarios (Teixeira et al., 2012; Wilkins, 2019). Para superar esta limitación, el artículo analiza la diversidad del sector privado, centrándose en examinar si la diferenciación de la oferta de formación refleja las estrategias que adoptan las IESp.

En la práctica, las tipologías disponibles tienen una aplicación limitada para evaluar el sector privado (Álvarez, 2013). Por una parte, porque la mayoría de estas asumen un perfil institucional de "talla única" (Sánchez-Barrioluengo, 2014), y no consideran como principio clasificatorio las estrategias de las universidades (Benneworth et al., 2016). Y por otra, porque tienden a ser tipologías estáticas que no reflejan las dinámicas de las IESp entre segmentos de mercado (Texeira et al. 2012; Wilkins, 2019), ni el surgimiento de nuevos productos y servicios académicos (Brunner, 2009). Para poder abordar este gap en la literatura, se clasifican las IESp introduciendo el concepto de grupos estratégicos que proviene de las teorías de Porter, y que es ampliamente discutido por Meilich (2019). Desde esta perspectiva, se desarrolla la idea de que un grupo estratégico agrupa a un conjunto homogéneo de IESp, que cuentan con ofertas de formación estructuradas de manera similar, y que logran tasas de matrícula similares, ya que persiguen estrategias similares. En este sentido, este artículo ofrece una primera reflexión sobre si el sector privado se segmenta en grupos que reflejan la adopción de estrategias comunes por parte de las IESp que los conforman o no.

Para lograr este objetivo se analizan las características que explican la segmentación del sector privado a través de un estudio empírico de las IESp colombianas. A su vez, se evalúa si la diferenciación de estas instituciones está vinculada con su perfil institucional o con las estrategias que persiguen. El caso colombiano constituye un escenario ideal para los objetivos del 
artículo, ya que en la última década Colombia ha pasado a ser uno de los países con mayor índice de privatización, según cifras publicadas por la Organización para la Cooperación y el Desarrollo Económico (OCDE, 2018).

En el artículo, se lleva a cabo la clasificación de las IESp aplicando un enfoque metodológico multidimensional y descriptivo (de La Torre et al., 2018). Se ha elegido esta alternativa metodológica porque permite establecer tipologías de IESp a partir de las diferencias o similitudes de las múltiples dimensiones que configuran la oferta de formación. Con ello, se espera contribuir a la literatura con la caracterización del sector privado desde una perspectiva estratégica. El hecho de adoptar el concepto de grupos estratégicos permite brindar información acerca de qué grupos son referentes en el sector (Ketchen et al., 2004), cuáles son las posibles vías de evolución de cada grupo, y qué tipo de barreras pueden influir en su evolución (Meilich, 2019). Los objetivos del artículo, por tanto, podrían ser de utilidad para los directivos del sector educativo en el diseño de sus estrategias organizacionales, así como para los gestores públicos en el diseño y/o mejora de las políticas que inciden sobre el desempeño y sostenibilidad de los sistemas de educación superior (Ketchen et al., 2004).

El artículo está organizado de la siguiente manera. La sección 2 presenta una revisión de la literatura sobre diversidad y diferenciación institucional en el sector privado. En la sección 3 se describen las especificaciones del análisis empírico que se lleva a cabo en el artículo. La sección 4 presenta los resultados del trabajo empírico, mientras que la sección final discute los resultados obtenidos a la luz del estado del arte.

\section{Diversidad institucional en el sector privado}

La diversidad institucional es definida como la variedad de IES que están presentes en un momento determinado en un sistema (en nuestro caso es el sistema de educación superior privado), en función de sus características institucionales (van Vught et al., 2010). La literatura ofrece diferentes aproximaciones metodológicas para medir la diversidad de los sistema de educación superior (Huisman et al., 2015). De entre ellas, las más reconocidas se relacionan con los procesos de diferenciación que ocurren en las IES (Rossi, 2009), en una dimensión horizontal o vertical (Daraio et al., 2011).

Un grupo estratégico se define como aquel subconjunto de entidades (en nuestro caso IESp) que cuentan con características similares y que se diferencian del resto de entidades/grupos que conforman la industria (i.e. en nuestro caso, el sector privado de la educación superior) (Meilich, 2019). 
Esta definición parte del supuesto de que la industria es heterogénea y está compuesta por grupos, a la vez asume que dichos grupos están conformados por entidades que siguen estrategias similares (Ketchen et al., 2004). Bajo esta lógica, parece razonable pensar que en el sector privado habrá grupos estratégicos en la medida en la que exista suficiente heterogeneidad en los mercados de consumo, así como en los productos o servicios académicos, como lo evidencian Dan et al., (2009), Duan (2019) y Wilkins (2019).

Convencionalmente, la distinción del sector privado de la educación superior se hace estrictamente en términos de la propiedad legal (Levy, 2013). En este sentido, la literatura se refiere a las IESp como entidades sin ánimo de lucro (aunque cada vez sean más los países que incentivan el surgimiento de IESp lucrativas), que son autónomas en la toma de decisiones y en su gobernabilidad (Raza, 2009). Estas instituciones emergen principalmente en entornos en los que, por un lado, el marco normativo les asigna un perfil institucional (p.e. carácter académico, calidad institucional, entre otros), y por otro, deciden autónomamente acerca de los segmentos sociales y económicos a los que están dirigidas (Levy, 2009). Los cambios demográficos, el crecimiento de un diversificado mercado laboral para graduados, y la emergencia y crecimiento de nuevas disciplinas han favorecido el incremento de la diferenciación de las IESp (Carpentier, 2018). Sin embargo, coexisten otras dinámicas en las que la competencia, la regulación y los intereses académicos generan comportamientos imitativos (Darraz y Bernasconi, 2012), condiciones que han sido desfavorables para la diversidad del sector privado debido a la eliminación de las IESp menos adaptables (Kwiek, 2017).

La literatura aborda la clasificación del sector privado desde dos enfoques (Álvarez, 2013). En el primero, y el más común, se comparan a las IESp conjuntamente con las instituciones públicas (p.e. Aldas et al., 2016). Estos trabajos señalan a las IESp como un grupo estratégico cuya diferenciación institucional está determinada principalmente por su especialización en la misión de la docencia (particularmente de posgrado) y la alta concentración en ciertas áreas de conocimiento. En el segundo enfoque, poco común, la literatura construye tipologías únicamente de las IESp. En este sentido, la clasificación de mayor referencia es la de Levy (2009) quien propone una tipología basada en la capacidad de las IESp para atraer y seleccionar estudiantes. A partir de su propuesta, se acuñaron los términos "universidades de élite" y "universidades no élite o de absorción de la demanda", refiriéndose en el primer caso, a las IESp que compiten tanto por estudiantes de élite como por académicos de prestigio. Por su parte, las universidades de "no élite" serían aquellas que tienden a ser poco selectivas en la admisión de estudiantes, y en las que en su oferta predominan los programas cortos y de bajo costo, sobre todo en áreas de ciencias sociales 
y administrativas. Sin embargo, cuando el propósito es evaluar el sector privado en un contexto particular, esta tipificación solo es orientativa, y por tanto, resulta necesario clasificar las IESp y revisar la efectividad y validez de los tipos identificados por la literatura (Álvarez, 2013).

\section{ANÁLISIS EMPÍRICO}

\section{El sector privado colombiano}

Históricamente, el sistema de educación superior colombiano ha sido mayoritariamente privado (Levy, 2013). La proporción del número de IESp frente a sus contrapartes del sector público fue, en 2018, del $71.1 \%$. En Colombia, el mayor número de IESp son de carácter universitario, reconocidas como Instituciones Universitarias/Escuelas Tecnológicas (IU de aquí en adelante) y Universidades, que en conjunto representan el 69\% del sistema.

El carácter académico y el tamaño organizacional son los factores comúnmente utilizados en la clasificación de las IES colombianas (Navas et al., 2020). De acuerdo con la normativa, la distinción entre IUs y Universidades tiene claras implicaciones en la forma en la que estas instituciones introducen y desarrollan su oferta académica. Por su parte, las Universidades deben incorporar de manera explícita la oferta de formación doctoral, estrechamente relacionada con las misiones de investigación y de extensión, mientras que las IUs pueden introducir estas misiones en la formación académica de manera gradual y con menores requerimientos. Este hecho ha derivado en que a lo largo de los años el número de universidades privadas se haya mantenido constante en el contexto colombiano, y que su expansión esté dirigida por la apertura de nuevas sedes. Por el contrario, las IUs se han duplicado en número, y han pasado a dominar el sector privado colombiano.

En el año académico 2018, 153 IESp estaban activas en Colombia. La muestra incluida en el presente análisis empírico está compuesta por 144 IESp que cumplen con los siguientes criterios: 1) son IES legalmente constituidas por sectores económicos o sociales independientes del Estado; 2) su carácter académico corresponde al de IUs y Universidades; 3) son IESp que cumplen con las tres misiones de la educación superior (docencia, investigación y extensión); 4) la oferta de formación dominante corresponde a programas académicos en los niveles 6, 7 y 8; 5) son IESp con registro vigente para la impartición de educación superior en el territorio colombiano, y cuentan con registros calificados vigentes (activos) para la impartición de al menos dos programas de pregrado universitario (nivel 
DORYS YANETH RODRIGUEZ CASTRO, JON MIKEL ZABALA-ITURRIAGAGOITIA, JUAN APARICIO

GRUPOS ESTRATÉGICOS EN EL SECTOR PRIVADO DE LA EDUCACIÓN SUPERIOR

6). Las estadísticas descriptivas de las instituciones colombianas están disponibles en el material adjunto (Anexo1).

\section{Diferenciación: variables e indicadores}

El artículo se centra en una dimensión de la diversidad, conocida como diferenciación horizontal, ya que describe la variación de aquellas actividades centrales de las IESp, que son ofrecidas a públicos destinatarios bien definidos (Daraio et al., 2011). El análisis se limita a caracterizar esta dimensión en términos de la oferta de formación existente en el sector privado y su relación con las tasas de matrícula, consideradas por la literatura como las dimensiones más adecuadas para evaluar la diversidad del sector privado (Álvarez, 2013). Para clasificar y caracterizar las IESp se seleccionan las variables directamente relacionadas con las estrategias aplicadas por estas, que son fuentes de diferenciación (Huisman et al., 2015; Rossi, 2009), y explican la cuota del mercado a la que están dirigidas (Brunner, 2009; Levy, 2009). La producción de la oferta académica doctoral y el número de estudiantes de doctorado son considerados indicadores de la participación de la IESp en investigación (Huisman et al., 2015; SánchezBarrioluengo, 2014). Las 25 variables utilizadas se presentan en la Tabla 1. Para hacer los resultados independientes de la escala de la medida, las variables son estandarizadas en una distribución con media de cero y varianza igual a 1 .

La producción de oferta académica se estima como la suma total del número de créditos académicos impartidos por año (Sav, 2016). Se ha optado por esta medida, frente a otras medidas utilizadas por la literatura, porque permite estandarizar el esfuerzo de la producción entre IESp, y además refleja la estrecha relación que existe entre los resultados, los requerimientos y los costos totales asociados a la impartición de los programas (ibid). La diversidad de la oferta de formación se describe en base a tres aspectos: niveles de formación, modalidades de impartición y campos de estudio. Para caracterizar la concentración en niveles de formación (incluida la modalidad de impartición) y el grado de especialización disciplinaria (campos de estudio) de las IESp se ha utilizado el índice GINI (Huisman et al., 2015; Rossi, 2009). Los valores de este indicador oscilan entre 0 y 1 , y cuando las IESp obtiene un valor cercano o igual a 0 se les denomina generalistas, debido a que la distribución disciplinaria o en niveles de formación tiende a ser similar a la distribución del sector privado en general.

El perfil institucional se ha definido a partir del carácter académico, la antigüedad, alcance territorial y el tamaño de la institución. Se han establecido tres rangos de antigüedad que discriminan entre IES pre1991, 
post1991, y antiguas (con más de 50 años) con respecto a la promulgación en 1991 de la Ley 30 de educación superior. El tamaño ha sido definido en base al número total de estudiantes matriculados y se han establecido cuatro rangos de tamaño: pequeño (menos de 5000 estudiantes), mediano (entre 5000 y 10000), grande (entre 10000 y 40000) y muy grande (más de 40000). El alcance territorial se describe respecto al tamaño y el desarrollo urbano de la ciudad donde se localización la IESp (ciudad grande, intermedia, pequeña) o si se localiza en varias ciudades (multicampus).

Se construyó una base de datos con las anteriores variables a partir del año 2014 y hasta diciembre de 2018. Se ha elegido este periodo temporal porque corresponde con la puesta en marcha de las políticas y estrategias para la masificación de la educación superior en Colombia (OECD, 2016). La información fue extraída de los informes públicos generados por el Sistema de Información Nacional para la Educación Superior (SNIES) administrado por el Ministerio de Educación Nacional (MEN) de Colombia. Para garantizar el manejo adecuado de la información institucional, las IESp son identificadas con el código asignado por el SNIES.

\section{Clasificación de las IESp}

El análisis empírico se realiza en dos etapas. Primero se aplica la técnica de escalamiento multidimensional (MDS) para probar si el sector privado colombiano es heterogéneo o no. El MDS es una técnica robusta para el análisis de variables con valores atípicos y variables redundantes. A su vez, no requiere cumplir con el supuesto de normalidad en los datos originales y los resultados que arroja no están influenciados por el número de variables (Borg et al., 2012)

EL MDS parte del supuesto de que las IESp que son similares tienden a ser cercanas en el espacio multidimensional resultante del mismo (Sagarra et al., 2015). Esto implica la reducción de la dimensionalidad de las 25 variables seleccionadas y la medición de las distancias entre las IESp. Para decidir cuál es el número $(m)$ de dimensiones que explica óptimamente el espacio multidimensional, se modelaron diferentes dimensiones en MDS hasta obtener el modelo de mejor ajuste, tomando como referencia el estadístico Stress-1. Las distancia entre las IESp se calculan mediante la distancia Euclídea, que es una medida apropiada para los propósitos del artículo porque identifica diferencias significativas entre dos IESp que están aisladas $\mathrm{y}$, a su vez, ignora las pequeñas diferencias que puedan existir entre IESp cercanas (Borg et al., 2012). A partir de estos resultados se generó una matriz simétrica de proximidades y otra de distancias que son utilizadas para configurar el espacio multidimensional. 
DORYS YANETH RODRIGUEZ CASTRO, JON MIKEL ZABALA-ITURRIAGAGOITIA, JUAN APARICIO GRUPOS ESTRATÉGICOS EN EL SECTOR PRIVADO DE LA EDUCACIÓN SUPERIOR

En este artículo se propone identificar los grupos bajo una lógica dual, es decir, que sean homogéneos internamente y heterogéneos entre sí. Con este objetivo se realizó un análisis de conglomerados sobre las coordenadas de las IESp en el espacio de m-dimensiones resultantes del MDS. Los grupos se forman aplicando el método de Ward, buscando maximizar la homogeneidad dentro de cada grupo y la heterogeneidad entre estos. El grado de distorsión entre el dendograma resultante y las relaciones originales entre las IESp se juzga a partir del coeficiente cofenético. Para corroborar si los grupos resultantes cumplen con la lógica dual propuesta, se aplicó un análisis de similitud (ANOSIM) a las distancias normalizadas entre IESp en el espacio configurado por las $m$-dimensiones. Utilizando el estadístico $\mathrm{R}$ se contrastó la hipótesis de la homogeneidad dentro de los grupos y de heterogeneidad entre grupos.

Para identificar cuáles son las variables que caracterizan los grupos resultantes se emplea el análisis de ajuste (Profitt). Este análisis consiste en una regresión lineal entre las coordenadas resultantes del MDS (eje$x)$ y las variables (eje-y) para cada IESp. Los coeficientes de regresión estandarizados $\left(\beta_{1 m}\right)$ permiten visualizar la posición, dirección y longitud de los vectores de las variables respecto a cada $m$-dimensión. Para probar si el perfil institucional explica el agrupamiento resultante, se realiza un análisis Profitt externo (Sagarra et al., 2015). Como indicadores del ajuste de la regresión se utilizan los estadísticos F y R2. Adicionalmente, se realiza un ANOVA y los estadísticos F y de Levene son utilizados para contrastar la hipótesis de que las diferencias entre las medias son explicadas por los grupos.

Para probar si los grupos resultantes representan grupos estratégicos, atendiendo a la definición ofrecida con anterioridad, se evaluó la relación entre las características distintivas de la oferta de formación y las tasas de matrícula. Para esto se aplicó un análisis de regresión lineal y se utilizaron los coeficientes de correlación como indicadores (valores cercanos a 1) de las características estratégicas en cada grupo. La validez estadística del coeficiente se evaluó utilizando el valor-p del estadístico F.

\section{RESULTADOS Y DISCUSIÓN}

En el sector privado colombiano el número de estudiantes matriculados por primera vez ha aumentado entre el año 2014 y 2018. En este periodo, las matrículas en posgrado aumentaron en aproximadamente un $25 \%$ y en pregrado universitario siguen mostrando una dinámica creciente (aumento del 7\%), tras la puesta en marcha del programa de becas para que estudiantes de escasos recursos estudien en universidades privadas acreditadas. No 
obstante, el número de estudiantes matriculados en programas técnicos y tecnológicos disminuyó en un 1\%, debido a la preferencia de muchos estudiantes colombianos a cursar programas profesionalizantes. En el mismo periodo, el número total de programas académicos aumentó en un $20 \%$, especialmente aquellos diseñados para impartir educación a distancia, que se duplicaron en número. No obstante, y aunque las tasas de crecimiento de la educación a distancia superan a las de la presencial, las cifras de otros países indican que la educación a distancia en Colombia podría crecer más rápido $(\mathrm{OECD}, 2016)$.

La Tabla 1 presenta las estadísticas descriptivas de la oferta de formación académica del sector privado colombiano para los años 2014 y 2018. Un primer análisis refleja que existen diferencias entre IUs y Universidades. En promedio, las Universidades tienden a tener un mayor número de estudiantes de pregrado y una oferta de formación más diversa que las IUs. Los resultados que arrojó el estadístico de Levene demostraron que el carácter académico se relaciona significativamente con la antigüedad y el tamaño de las IESp (número de estudiantes matriculados), que son comúnmente utilizados por la literatura para caracterizar el perfil institucional. Las estadísticas también muestran que existen diferencias significativas en el número de estudiantes de posgrado, programas académicos y la producción de créditos académicos. Sin embargo, se debe ser cuidadoso en atribuirle estas diferencias al carácter académico, porque en estos casos la varianza agregada es mayor que la varianza total (Tabla 1). Estos resultados permiten concluir que los datos agregados por el carácter académico pueden enmascarar dinámicas de diferenciación institucional, $\mathrm{y}$, por tanto, no permiten visualizar la existencia de otros factores que son claves en la clasificación de las IESp.

Tabla 1

Variables y estadísticas descriptivas

\begin{tabular}{|c|c|c|c|c|c|c|}
\hline \multirow[t]{2}{*}{ Aspecto } & & \multirow[t]{2}{*}{$\begin{array}{l}\text { Descripción de las va- } \\
\text { riables }\end{array}$} & \multirow[t]{2}{*}{$\begin{array}{l}\text { Med. } \\
\text { Desv. }\end{array}$} & & \multicolumn{2}{|c|}{$\begin{array}{l}\text { Comparación } \\
\text { Carácter aca- } \\
\text { démico }\end{array}$} \\
\hline & & & & & Anova & Levene \\
\hline \multirow{4}{*}{$\begin{array}{l}\text { Perfil Insti- } \\
\text { tucional }\end{array}$} & & Carácter académico & & & & \\
\hline & & Alcance territorial & & & & \\
\hline & & Año de creación & & & & \\
\hline & V1 & $\begin{array}{l}\text { Número de años de anti- } \\
\text { güedad con corte a julio de } \\
2019\end{array}$ & 36.0 & 16.9 & $.000 * *$ & $.800^{\mathrm{a}}$ \\
\hline
\end{tabular}




\begin{tabular}{|c|c|c|c|c|c|c|}
\hline \multirow[t]{2}{*}{ Aspecto } & & \multirow[t]{2}{*}{$\begin{array}{l}\text { Descripción de las va- } \\
\text { riables }\end{array}$} & \multirow[t]{2}{*}{$\begin{array}{l}\text { Med. } \\
\text { Desv. }\end{array}$} & & \multicolumn{2}{|c|}{$\begin{array}{l}\text { Comparación } \\
\text { Carácter aca- } \\
\text { démico }\end{array}$} \\
\hline & & & & & Anova & Levene \\
\hline \multirow{6}{*}{$\begin{array}{l}\text { Tamaño } \\
\text { 2014-2017 }\end{array}$} & $\mathrm{V} 2$ & $\begin{array}{l}\text { Número de estudiantes ma- } \\
\text { triculados Total }\end{array}$ & 7769.2 & 12463.2 & $.000 * *$ & $.633^{\mathrm{a}}$ \\
\hline & V3 & Pregrado & 6987.8 & 11735.5 & $.002 * *$ & $.782^{\mathrm{a}}$ \\
\hline & V4 & Posgrado & 781.6 & 1440.9 & $.000 * *$ & .000 \\
\hline & V5 & $\begin{array}{l}\text { Número de programas To- } \\
\text { tal }\end{array}$ & 24.0 & 55.7 & $.000 * *$ & .000 \\
\hline & V6 & Pregrado & 16.0 & 21.1 & $.000 * *$ & $.270^{\mathrm{a}}$ \\
\hline & V7 & Posgrado & 12.0 & 44.4 & $.000 * *$ & .000 \\
\hline \multirow{9}{*}{$\begin{array}{l}\text { Producción } \\
\text { Presencial } \\
(2014-2017)\end{array}$} & V8 & $\begin{array}{l}\text { Suma total Créditos acadé- } \\
\text { micos presencial }\end{array}$ & 1515.0 & 1714.2 & $.000 * *$ & .000 \\
\hline & V9 & Pregrado (excepto salud) & 527.6 & 541.0 & $.000 * *$ & .000 \\
\hline & V10 & Pregrado, salud & 104.9 & 83.5 & $.001 * *$ & .000 \\
\hline & V11 & $\begin{array}{l}\text { Especializaciones universi- } \\
\text { tarias }\end{array}$ & 419.8 & 609.4 & $.000 * *$ & .000 \\
\hline & V12 & Maestrías & 369.2 & 429.1 & $.000 * *$ & .000 \\
\hline & V13 & Especializaciones salud & 746.5 & 914.2 & $.001 \% *$ & .000 \\
\hline & V14 & Doctorado & 105.5 & 99.5 & $.000 * *$ & .000 \\
\hline & V15 & Técnicos & 113.3 & 148.7 & .364 & $.043^{\mathrm{a}}$ \\
\hline & V16 & Tecnológicos & 167.0 & 161.6 & .180 & $.038^{\mathrm{a}}$ \\
\hline \multirow{5}{*}{$\begin{array}{l}\text { Producción } \\
\text { a distancia } \\
(2014-2017)\end{array}$} & V17 & $\begin{array}{l}\text { Créditos académicos Téc- } \\
\text { nicos }\end{array}$ & 91.0 & 64.3 & .374 & $.059^{\mathrm{a}}$ \\
\hline & V18 & Tecnológicos & 99.8 & 74.0 & .631 & $.234^{\mathrm{a}}$ \\
\hline & V19 & Pregrado todos los campos & 148.4 & 126.2 & .657 & $.707^{\mathrm{a}}$ \\
\hline & V20 & $\begin{array}{l}\text { Especializaciones universi- } \\
\text { tarias }\end{array}$ & 96.6 & 85.6 & 0.025 & $.237^{\mathrm{a}}$ \\
\hline & V21 & Maestrías & 76.0 & 72.3 & $.000 * *$ & .000 \\
\hline \multirow{4}{*}{$\begin{array}{l}\text { Especializa- } \\
\text { ción (Índice } \\
\text { GINI) y Di- } \\
\text { versidad } \\
\text { (Índice } \mathrm{H}^{\prime} \text { ) }\end{array}$} & V22 & $\begin{array}{l}\text { Especialización Oferta de } \\
\text { formación }\end{array}$ & .9 & .1 & $.000 * *$ & $.753^{\mathrm{a}}$ \\
\hline & V23 & $\begin{array}{l}\text { Diversidad Oferta de for- } \\
\text { mación }\end{array}$ & 4.0 & 2.8 & $.000 * *$ & $.119^{\mathrm{a}}$ \\
\hline & V24 & Especialización disciplinar & .9 & .1 & $.000 * *$ & $.949^{\mathrm{a}}$ \\
\hline & V25 & Diversidad disciplinar & 7.0 & 4.2 & $.000 * *$ & $.746^{\mathrm{a}}$ \\
\hline
\end{tabular}

a Indica la homogeneidad de varianzas. ** La diferencia es significativa al nivel de .005

Fuente de datos: Elaboración propia 
Para probar si el sector privado colombiano es heterogéneo se decide modelar un MDS para espacios conformados entre 1-dimensión y hasta 7-dimensiones. El estadístico de Stress-1 disminuye gradualmente hasta encontrar en los modelos 6-dimensión y 7-dimensión los valores más bajos. Entre los dos se optó por descartar el modelo 7-dimensión porque el valor del estadístico Stress-1 incrementa ligeramente. La medida de bondad y ajuste del modelo de 6 dimensiones es igual a .05, considerado como excelente en la clasificación de Kruskal (Borg et al., 2012) y por tanto, se estima que es una solución óptima para representar la heterogeneidad del sector privado colombiano.

A continuación, se examina si existen grupos de IESp en el espacio de 6-dimensiones aplicando un análisis de conglomerados. Para conocer cuáles de estas instituciones tienden a agruparse por su cercanía, se utilizan como medidas de referencia a las seis coordenadas que posicionan a cada IESp en el MDS (ver Anexo1). El resultado de este análisis arrojó el agrupamiento de las IESp en cinco grupos (Figura 1). Inicialmente se identifican cuatro grupos (distancia de 4). Sin embargo, el grupo de mayor tamaño se subdivide en dos grupos a una menor distancia. A partir del coeficiente cofenético (valor .82) se concluyó que el dendograma representa una solución óptima para caracterizar los grupos resultantes, y al comparar el grado de similitud existente entre grupos, se comprobó que son distintos $(\mathrm{R}=.71)$. A partir de este resultado, se opta por seleccionar el modelo de 5 grupos, ya que el rango medio entre grupos es significativamente mayor que dentro de los grupos. Este resultado apoya la idea de que los cinco grupos resultantes responden a la lógica dual que se requiere para establecer grupos estratégicos. 
DORYS YANETH RODRIGUEZ CASTRO, JON MIKEL ZABALA-ITURRIAGAGOITIA, JUAN APARICIO

Figura 1

Dendograma

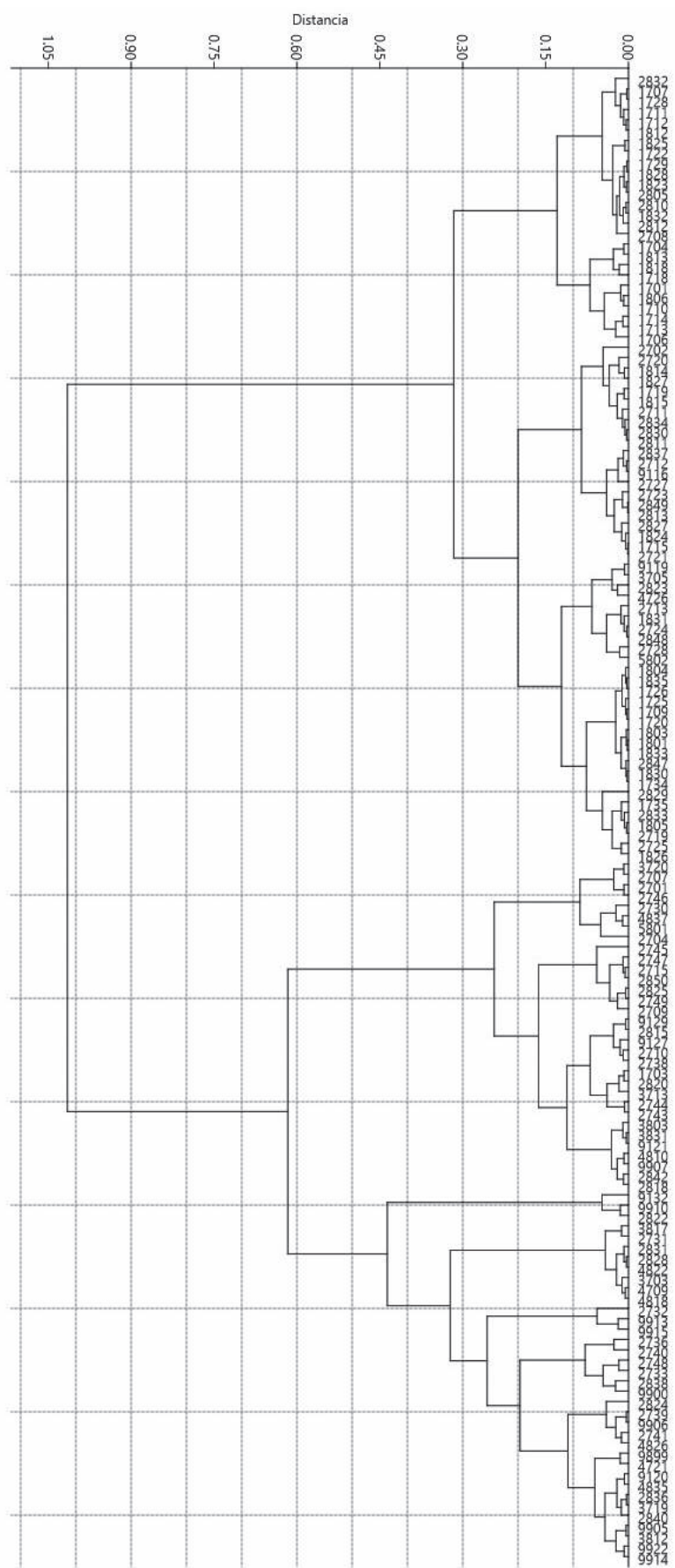


Los grupos identificados responden a un perfil institucional común. La caracterización de los grupos según el carácter académico, la antigüedad, el alcance territorial y el tamaño del conjunto de IESp que los conforman arrojó los siguientes perfiles. El primer Grupo (C1) reúne a las universidades más antiguas, mayoritariamente multicampus y de tamaño grande/mediano. El segundo (C2), agrupa seis IUs, incluyendo a las tres más grandes y de mayor trayectoria del país (pre/1991). El tercer Grupo (C3) está conformado por IUs y Universidades medianas/pequeñas con trayectoria en el sector (pre/1991) y ubicadas en ciudades intermedias o pequeñas. El cuarto grupo se subdivide en dos grupos. El primer subgrupo (C4) reúne a las IUs más pequeñas del sector localizadas en ciudades grandes, mientras que el segundo subgrupo (C5), incluye a las IUs y algunas universidades pequeñas con trayectoria en el sector (pre/1991) localizadas mayoritariamente en ciudades pequeñas.

Un primer análisis del MDS arrojó que la proximidad de las IESp en la dimensión 1 está definida principalmente por el tamaño, ubicando en el lado derecho a las IESp pequeñas y en el lado izquierdo a las instituciones de mayor tamaño (Figura 2). Sin embargo, al comparar el grado de similitud entre grupos, el resultado del ANOSIM muestra que hay unos más próximos que otros (Tabla 2). Por una parte, se evidencia que los grupos que incluyen a las IESp medianas y pequeñas (Grupos 3, 4 y 5) son próximos, y que esta proximidad es independiente del carácter académico de las instituciones que los conforman. Por otra, se evidencia que los dos grupos que incluyen a las IESp de mayor tamaño (Grupos 1 y 2) se diferencian entre sí, y también del resto de los grupos, de manera que las Universidades tienden a diferenciarse de las IUs cuando se trabaja a mayores escalas de matrícula. Desde la perspectiva de grupos estratégicos adoptada en el artículo, este resultado tiene dos implicaciones. La primera es que la escala de trabajo representa uno de los factores clave en la clasificación de las instituciones colombianas, como lo anuncia Navas et al. (2020), y la segunda, que el carácter académico puede llegar a ser una barrera para la evolución de las IESp que operan a mayores escalas (Meilich, 2019). En otras palabras, podría decirse que la división del trabajo entre Universidades y no Universidades es evidente entre las IESp de mayor tamaño, mientras que en IESp medianas y pequeñas los límites parecen ser borrosos, similar a como ocurre en otros países emergentes (Arimoto, 2014). 
DORYS YANETH RODRIGUEZ CASTRO, JON MIKEL ZABALA-ITURRIAGAGOITIA, JUAN APARICIO

Tabla 2

Análisis de similaridad

\begin{tabular}{lllllll}
\hline & & \multicolumn{5}{c}{ Estadístico $R$} \\
\cline { 3 - 7 } & & $\mathrm{C} 1$ & $\mathrm{C} 2$ & $\mathrm{C} 3$ & $\mathrm{C} 5$ & $\mathrm{C} 4$ \\
& NoIESp & 13 & 6 & 33 & 59 & 33 \\
& $\mathrm{C} 1$ & & .757 & .804 & .925 & .981 \\
Bonferroni & $\mathrm{C} 2$ & .002 & & .949 & .974 & .993 \\
Sig. & $\mathrm{C} 3$ & .001 & .001 & &. $\mathbf{3 9 6}^{\mathbf{a}}$ & .859 \\
& $\mathrm{C} 5$ & .001 & .001 & .001 & & $\mathbf{. 5 7 4}^{\mathbf{a}}$ \\
& $\mathrm{C} 4$ & .001 & .001 & .001 & .001 & \\
\hline
\end{tabular}

a Alta similitud

Figura 2

Distribución del Perfil Institucional en MDS

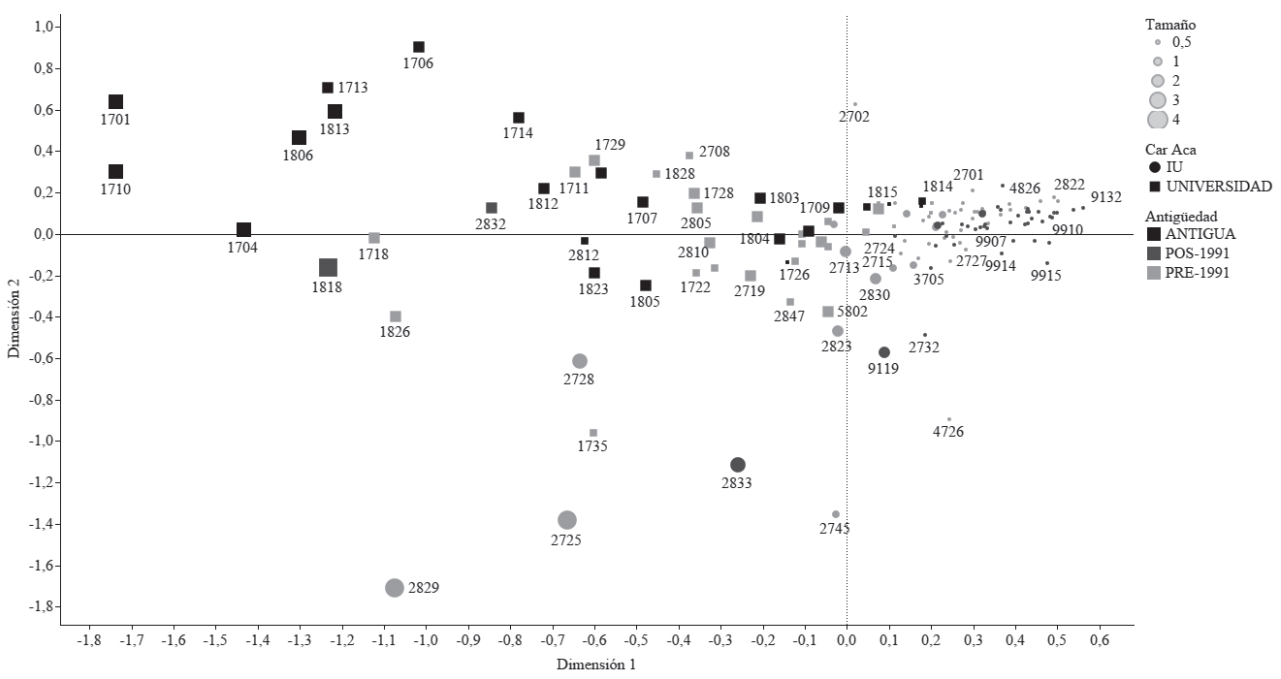

Con el fin de entender cuáles son las características de la oferta de formación que diferencia a los grupos y los diversos tipos de IESp, se recurre al análisis Profitt (Anexo1). Este análisis encaja el vector que representa cada variable a través del espacio 6-dimensiones, de tal manera que una característica particular de los IESp crece en la misma dirección del vector. Para facilitar su interpretación se han representado los vectores en las dos primeras dimensiones y diferenciado a las IESp por Grupo (Figura 3). 
Figura 3

Grupo de IESp en el espacio multidimensional (MDS)

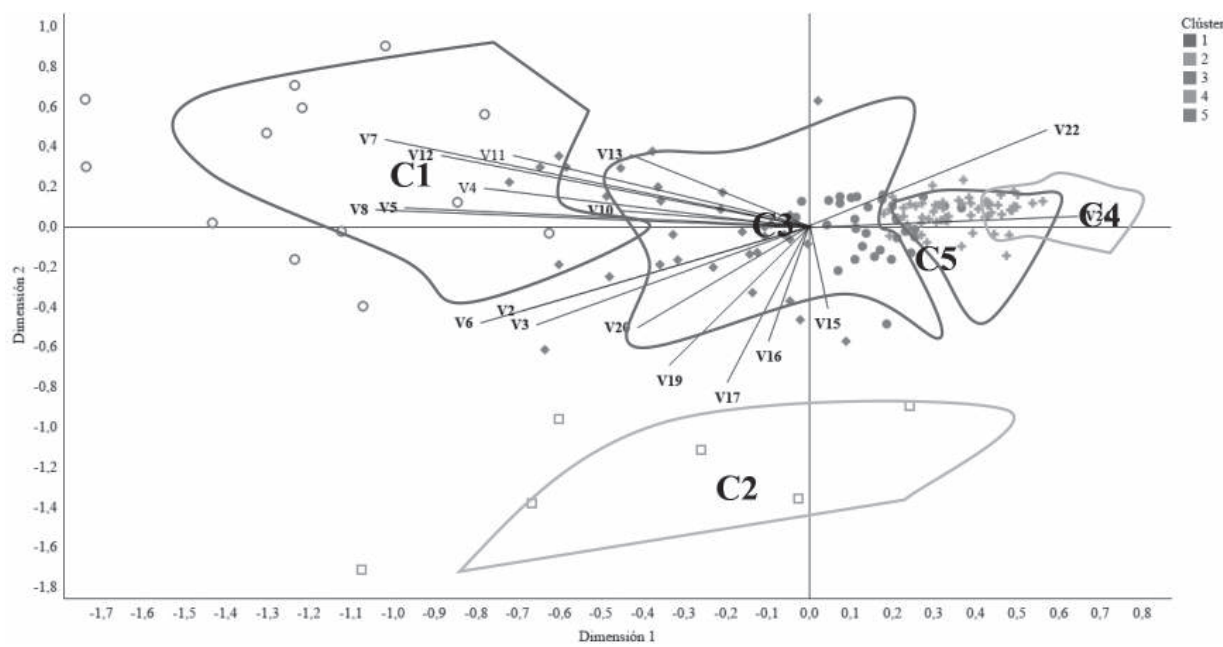

La distribución de las IESp en la primera dimensión está determinada principalmente por dos factores. El primer factor corresponde al volumen en la producción de oferta académica, explicado en términos del número total de programas y la suma total de créditos académicos, ubicando en el lado izquierdo a IESp que ofertan el mayor número de programas y que tienen una alta actividad de producción en créditos académicos al año. El segundo factor es el grado de especialización en la oferta de formación, encontrando en el lado derecho de esta dimensión a las IUs especializadas en pocos niveles de formación y/o en un solo campo de estudio. Contrariamente, en el lado izquierdo se encuentran las Universidades con una producción académica diversa tanto en niveles de formación, como en campos de estudios. Esto sugiere que la Dimensión 1, puede ser etiquetada como el volumen de la oferta de formación vs su especialización. Analizando la segunda dimensión (Figura 2 ), cabe mencionar que en la parte inferior están ubicadas las instituciones con una mayor oferta de formación técnica y tecnológica, tanto de manera presencial como a distancia. Las IESp que se encuentran aquí (Grupo 2), son entre otras las IUs de mayor tamaño y crecimiento en educación a distancia. En la parte superior de esta dimensión se ubican principalmente las Universidades que imparten mayoritariamente la formación posgradual, particularmente especializaciones universitarias y maestrías (Grupos 1 y 3). Esto sugiere que la Dimensión 2, puede ser interpretada como orientada hacia la masificación de la formación técnica y tecnológica vs la formación en posgrado.

Para poder examinar si la diferenciación de la oferta de formación refleja las estrategias que los grupos identificados adoptan para competir por 
estudiantes, se correlacionan la variación del número de programas frente a la variación en las tasas de matrícula para cada grupo. En total fueron realizadas tres regresiones, utilizando las tasas de matrícula total (regresión 1), de pregrado (regresión 2) y de posgrado (regresión 3). Los resultados que arrojan estas regresiones permiten identificar las estrategias que adoptan los grupos para atraer a más estudiantes (Tabla 3). La regresión realizada para la matrícula total dejó en evidencia tres estrategias. La primera estrategia se centra en aumentar y consolidar la oferta de programas de posgrados, lo cual afecta positiva y significativamente las tasas de matrícula total de las IESp incluidas en el Grupo 1. A partir del coeficiente de correlación con las tasas de matrícula de posgrado, se concluye que esta estrategia se relaciona principalmente con el aumento de la oferta de programas de maestría. A pesar de que el aumento en el número de especializaciones universitarias, doctorados y especializaciones médicas también contribuyen al crecimiento de las matrículas, esta relación baja.

Tabla 3

Coeficientes de regresión

\begin{tabular}{|c|c|c|c|c|c|c|}
\hline Dimensión & Programas & C1 & $\mathrm{C2}$ & C3 & C4 & C5 \\
\hline \multirow[t]{10}{*}{ MAT_Total } & N5-2018 & -.727 & -.255 & $.581 * *$ & .148 & .148 \\
\hline & N5-2010 & -.383 & $.914 * * *$ & .267 & .024 & -.044 \\
\hline & N6-2018 & -.16 & .598 & $.468 * *$ & .122 & -.061 \\
\hline & N6-2010 & -.357 & $.788 * *$ & -.02 & -.275 & -.115 \\
\hline & N71-2018 & .1 & .409 & -.118 & -.093 & .012 \\
\hline & N71-2010 & -.385 & -.055 & .042 & .174 & .199 \\
\hline & N72y8-2018 & $.604 * *$ & .253 & -.171 & -.157 & .057 \\
\hline & N72y8_2010 & $.747 * * *$ & $.805 * *$ & -.176 & -.023 & $.304 *$ \\
\hline & Distancia-2018 & .045 & -.296 & .168 & $.416 \% *$ & $.279 *$ \\
\hline & Distancia-2010 & .091 & $.678 *$ & .068 & -.003 & .064 \\
\hline \multirow[t]{6}{*}{ MAT_PREG } & N5-2018 & -.67 & -.259 & $.573 * *$ & .153 & .158 \\
\hline & N5-Total & -.614 & .102 & $.588 * *$ & .141 & .116 \\
\hline & N6-2018 & -.166 & .597 & $.438 * *$ & .112 & -.055 \\
\hline & N6-Total & -.461 & $.845 * *$ & .285 & -.173 & -.163 \\
\hline & Distancia5y6-2018 & .072 & -.3 & .24 & $.42 * * *$ & $.26 *$ \\
\hline & Distancia5y6-Total & .081 & .125 & .241 & $.38 * * *$ & $.215 *$ \\
\hline \multirow[t]{3}{*}{ MAT_POSG } & N71-2018 & $.398 *$ & $.647 * *$ & -.023 & $.271 * *$ & $.357 * *$ \\
\hline & N71-Total & .223 & -.018 & .11 & $.377 * * *$ & $.412 * * *$ \\
\hline & N73-Total & .317 & -.698 & .031 & .017 & . \\
\hline
\end{tabular}




\begin{tabular}{|c|c|c|c|c|c|c|}
\hline Dimensión & Programas & C1 & $\mathrm{C} 2$ & C3 & C4 & C5 \\
\hline & N72-2018 & $.721 * * *$ & .509 & .017 & -.353 & .217 \\
\hline & N72-Total & $.705 * * *$ & .545 & .005 & -.283 & .26 \\
\hline & N8-2018 & $.381 *$ & .143 & -.315 & $.2 *$ & .153 \\
\hline & N8-Total & $.52 * *$ & .24 & -.296 & $.2 *$ & .153 \\
\hline
\end{tabular}

Nivel de significancia: $* * * .005 ; * * .05 ; * .1$

La segunda estrategia está dirigida a consolidar la oferta de formación de pregrado, lo cual afecta positiva y significativamente las tasas de matrícula total de las IESp incluidas en los Grupos 2 y 3. Por una parte, el aumento del número de programas técnicos, tecnológicos y de pregrado universitario contribuyen significativamente al incremento de las matrículas de las IESp del Grupo 3. Por otra, la consolidación de la oferta de programas de pregrado universitario presencial o a distancia sustenta el crecimiento de las matrículas en las IESp incluidas en el Grupo 2, que son las de mayor tamaño.

Por último, la tercera estrategia está relacionada con el incremento en la oferta de formación a distancia que ocurre en los Grupos 4 y 5 . El aumento de programas técnicos, tecnológicos y de pregrado universitarios impartidos en la modalidad a distancia ha favorecido significativamente el crecimiento de las tasas de matrícula en las IESp incluidas en el Grupo 4, y en menor grado en las IESp incluidas en el Grupo 5. Por otra parte, el aumento en el número de programas de pregrado impartidos presencialmente no afecta positiva y significativamente al aumento de las matrículas en las IESp de los Grupo 4 y 5.

La identidad de los grupos estratégicos propuesta es la siguiente: El primer Grupo agrupa 13 Universidades. Son las más antiguas y las de mayor tamaño. La identidad estratégica de este grupo está fuertemente orientada a la atracción de estudiantes de posgrado en la modalidad presencial. Su oferta de formación está dominada por programas de posgrado, en particular de maestrías y especializaciones universitarias. Cuenta con la mayor oferta de formación doctoral, y por tanto se concluye que su oferta de formación está sustentada en el desarrollo de la misión de investigación. En el periodo 20142018 la dinámica de crecimiento en la oferta de formación de posgrados está relacionada con el aumento en el número total de estudiantes. Consecuentemente, el número de estudiantes en estos niveles de formación es muy superior al del resto de grupos. 
DORYS YANETH RODRIGUEZ CASTRO, JON MIKEL ZABALA-ITURRIAGAGOITIA, JUAN APARICIO GRUPOS ESTRATÉGICOS EN EL SECTOR PRIVADO DE LA EDUCACIÓN SUPERIOR

El Grupo 2 reúne a las IUs con el mayor número de estudiantes matriculados, y que están radicadas en ciudades grandes. La característica distintiva de este grupo es la formación de pregrado, sustentada principalmente por la formación profesional universitaria. Este grupo también muestra los más altos niveles de actividad en la producción de oferta académica para la formación técnica y tecnológica, tanto en la modalidad presencial como a distancia. Sin embargo, debido a que la atracción de nuevos estudiantes de pregrado es fruto de la oferta consolidada antes del 2010, se considera que no forma parte de la identidad estratégica actual. Por otro lado, aunque la formación posgradual es incipiente, la dinámica de crecimiento en el número de especializaciones universitarias apunta a que esta es una de las estrategias de crecimiento en matrícula que están poniendo en marcha las IES incluidas en este Grupo.

El Grupo 3 está compuesto por 33 IESp. Agrupa mayoritariamente Universidades, y algunas IUs, de tamaño mediano/pequeño que se localizan principalmente en ciudades intermedias y pequeñas. Respecto a los otros grupos, la característica distintiva de este grupo es la formación de pregrado, sustentada tanto en programas profesionalizantes como en programas para la formación técnica y tecnológica. Sin embargo, llama la atención que el número de estudiantes de posgrado ha aumentado en los últimos años mientras que la oferta de programas se ha mantenido estable, lo que implica una mayor intensidad en la frecuencia de impartición de los programas, especialmente en aquellos de ciclos cortos como lo son las especializaciones universitarias. Este hallazgo saca a la luz otra posible estrategia de crecimiento, que consiste en alcanzar una mayor relación del número de alumnos por programa al año.

El cuarto Grupo está compuesto por 59 IESp. Todas estas son IUs de tamaño pequeño. La mayoría de ellas son jóvenes (pos/1991) y están localizadas en ciudades grandes. Cuentan con una producción académica baja y poco diversa. La orientación estratégica de este grupo apunta a la educación a distancia. El aumento en el número de programas tanto de pregrado como de especializaciones universitarias impartidos bajo esta modalidad ha favorecido el crecimiento de la matrícula en las IESp incluidas en este Grupo. También hay que destacar el crecimiento en el número de las especializaciones médicas, aunque su relación con el aumento del número de estudiantes de posgrado es baja. La formación posgradual es incipiente, especialmente aquella que demanda altos niveles de exigencia en la condición de investigación (no cuenta con programas de doctorado).

El Grupo 5 agrupa 33 IESp. Son IUs y algunas Universidades pequeñas con trayectoria (pre/1991), en su mayoría de carácter regional. Este grupo se identifica porque la oferta de formación en áreas de la salud 
es incipiente. Aunque los niveles de producción académica posgradual son bajos, la estrategia de crecimiento está dirigida por el aumento en la oferta de especializaciones universitarias y maestrías. En el periodo analizado, el número de estudiantes matriculados en pregrado ha disminuido pese a los esfuerzos en aumentar la oferta de educación a distancia. Por esta razón, se toma la decisión de no considerar esta relación como una estrategia de atracción de estudiantes.

Adoptar el concepto de grupo estratégico para el manejo del sector privado de la educación superior puede resultar de gran utilidad. Particularmente, es útil para mejorar las políticas e instrumentos de coordinación del sistema de educación superior, ya que ayuda en la identificación del rol de cada grupo en su sostenibilidad o competitividad, facilitando la definición de objetivos de política diferenciados y centrados. Como se muestra en la Figura 4, la distribución y las características de los grupos identificados en el caso colombiano conllevan a que los gestores de la política de educación y ciencia se planteen diferentes rutas o alternativas de crecimiento para cada uno de estos. Por ejemplo, el grupo C4 (Grupo 4) conformado principalmente por las IUs más jóvenes, tiene al menos tres alternativas para crecer y desarrollarse. Del mismo modo, las Universidades tradicionales C3 (Grupo 3), que al parecer están detenidas en su crecimiento, o las Universidades del C1 (Grupo 1), que al parecer se limitan a atender el mercado nacional, podrían seguir rutas diferenciadas. A partir de esta ilustración se concluye que la identificación de grupos estratégicos como los obtenidos en esta investigación brindan información valiosa para generar escenarios de crecimiento y competitividad de un sistema de educación superior.

Figura 4

Posibles rutas de avance y alternativas de crecimiento para los grupos de IESp identificados

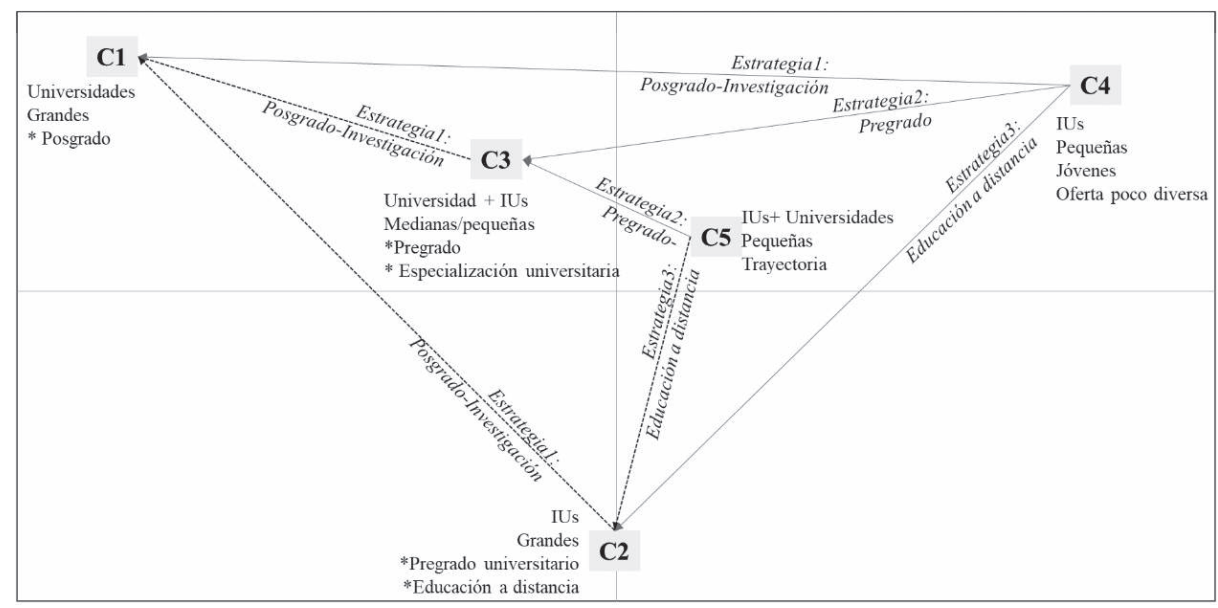


DORYS YANETH RODRIGUEZ CASTRO, JON MIKEL ZABALA-ITURRIAGAGOITIA, JUAN APARICIO

\section{CONCLUSIONES}

En este artículo se han identificado cinco tipologías de IESp que son considerados como "grupos estratégicos", ya que agrupan a las instituciones que tienden a estructurar su oferta de formación de modo similar desde un punto de vista estratégico (Meilich, 2019). A su vez, se ha evidenciado que la educación a distancia y la formación técnica y tecnológica son fuentes relevantes de diferenciación en el sector privado, incluso en casos como el colombiano, donde el desarrollo de estos mercados es bastante marginal (OECD, 2016). En este sentido, a pesar de existir un marco regulatorio común, las IESp colombianas no solo son diversas en términos de su perfil institucional, sino que además esta investigación demuestra que también son heterogéneas en sus intereses de mercado. Esto probablemente conduce a que existan diferentes estructuras para la producción de oferta académica (como lo evidencia Duan, 2019), a las cuales es necesario prestar mayor atención en las evaluaciones de desempeño del sector privado, pudiendo convertirse en un campo interesante para futuras investigaciones.

Otros factores determinantes de la diversidad del sector privado son el tamaño de las IESp y la oferta de programas que requieren cumplir con mayores requisitos de investigación (formación doctoral). En la tipología que se construye en el artículo, solo los grupos 1 y 2, conformados principalmente por Universidades grandes, tienen una oferta doctoral importante. Esto puede sugerir que la diferenciación institucional de las IESp podría implicar economías de alcance en la docencia, y estas a su vez, en el desarrollo de las misiones de investigación y tercera misión (De Witte y Hudrlikova, 2013), a las cuales también es necesario prestar atención en las evaluaciones de desempeño del sector privado.

El alcance territorial juega un papel clave en la definición de los grupos estratégicos en el sector privado colombiano. Claramente, los grupos estratégicos que lideran en el sector de la educación superior privada en Colombia (grupos 1 y 2) están ubicados en la ciudad capital (de mayor tamaño y desarrollo urbano) y se han extendido al resto del país a través de sedes multicampus. Por su parte, en las regiones se encuentran Universidades e IUs que a lo largo de los años mantienen su enfoque estratégico en el pregrado (grupos 3 y 5). En otras palabras, el alcance territorial es una fuente relevante de diferenciación, y no considerarlo al analizar el desempeño de las IESp podría tener consecuencias no deseadas para el sistema de educación superior. Además, desconocer el alcance territorial implicaría ignorar parte de la contribución de estas instituciones al desarrollo regional (no reflejado en las clasificaciones universitarias actuales) y podría llegar a penalizar, particularmente, a aquellas IESp que orientan la tercera misión 
a este propósito (Benneworth et al., 2016), convirtiéndose en un campo interesante para futuras investigaciones.

A pesar de que Navas et al. (2020) utilizan la misma información que nuestro estudio, llama la atención que los cinco grupos que identificaron incluyen IES públicas y privadas, y que las IES privadas no constituyan un grupo estratégico aparte, como sí ocurre en estudios similares (p.e. Aldas et al., 2016; Sagarra et al., 2015). En este sentido, nuestra investigación proporciona resultados más orientados al manejo del sector privado, gracias a la solidez de la técnica MDS y a la minuciosa caracterización de las fuentes de diversidad de este sector.

\section{NOTAS}

1 Los principales segmentos de mercado son los programas de pregrado, de posgrados profesionales, de grados avanzados de investigación (doctorado), educación continua, educación a distancia, formación para empresas y formación para la investigación (Brunner, 2009).

2 La normativa colombiana clasifica a las IESp en cuatro tipos, según su carácter académico: Las instituciones técnicas profesionales y tecnológicas son excluidas de esta investigación debido a que su oferta de formación se centra en programas técnicos/tecnológicos y de corta duración. Además, su funcionamiento para cumplir con las misiones de investigación y extensión es marcadamente inferior respecto a las IUs y universidades.

3 Los niveles de formación corresponden a: Educación técnica profesional y tecnológica (nivel 5); Pregrado universitario con duración igual o superior a los 4 años (nivel 6); Especializaciones universitarias y Maestrías (nivel 7) y Doctorado (nivel 8) (DANE, 2011).

4 Los programas académicos se clasificaron en función del nivel de formación y el campo de estudio utilizando la nomenclatura descrita (DANE, 2011).

5 El crédito académico se refiere a la unidad de medida de la carga académica de un programa académico (Sav, 2016). 
DORYS YANETH RODRIGUEZ CASTRO, JON MIKEL ZABALA-ITURRIAGAGOITIA, JUAN APARICIO

\section{REFERENCIAS BIBLIOGRÁFICAS}

Aldas, J., Escribá, A., Iborra, M., y Safón, V. (2016). La Universidad Española: Grupos estratégicos y desempeño. Fundación BBVA.

Álvarez, G. (2013). Tipologías de las instituciones de educación superior privadas ¿para qué? En J. Silas (Ed.), Estados de la educación superior en America Latina, el balance públicoprivado. ITESO.

Arimoto, A. (2014). The Teaching and Research Nexus in the Third Wave Age. In J., Shin, A. Arimoto, W. Cummings, \& U. Teichler. (Eds.), Teaching and Research in Contemporary Higher Education (pp. 15-35).

Benneworth, P., Pinheiro, R., \& SánchezBarrioluengo, M. (2016). One size does not fit all! New perspectives on the university in the social knowledge economy. Science and Public Policy, 43(6), 731-735, https://doi.org/10.1093/ scipol/scw018

Borg, I., Groenen, P., \& Mair, P. (2012). Applied Multidimensional Scaling, Springer. https://doi.org/10.1007/97881-322-0763-4

Brunner, J. (2009). Educación Superior en Chile: Instituciones, mercados y politicas gubernamentales, 1967-2007. [Tesis doctoral]. Leiden University.

Carpentier, V. (2018). Expansion and differentiation in higher education: the historical trajectories of the UK, the USA and France. Center for Global Higher Education.

Dan, M., Ritchie, W., \& Geiger, S. (2009). Strategic Group Membership and Nonprofit Organization Performance. Nonprofit Management and Leadership, 20(1), 23-39.
DANE (2011). Clasificación Internacional Normalizada de la Educación adaptada para Colombia CINE 2011.

Daraio, C., Bonaccorsi, A., Geuna, A., Lepori, B., Bach, L., Bogetoft, P., ... Eeckaut, \& P. Vanden. (2011). The European university landscape: A micro characterization based on evidence from the Aquameth project. Research Policy, 40(1), 148-164

Darraz, E. y Bernasconi, A. (2012). Elementos conceptuales para el análisis organizacional de universidades en contextos de mercado. Innovar, 22(46), 87-97.

de La Torre, E., Casani, F., \& Sagarra, M. (2018). Defining typologies of universities through a DEAMDS analysis: An institutional characterization for formative evaluation purposes. Research Evaluation, 27(4), 388-403.

De Witte, K. \& Hudrlikova, L. (2013). What about excellence in teaching? A benevolent ranking of universities. Scientometrics, 96, 337-364.

Duan, S. (2019). Measuring university efficiency: An application of data envelopment analysis and strategic group analysis to Australian universities. Benchmarking, 26(4), 1161-1173.

Huisman, J., Lepori, B., Seeber, M., Frølich, N., \& Scordato, L. (2015). Measuring institutional diversity across higher education systems. Research Evaluation, 24(4), 369-379.

Ketchen, D., Snow, C., \& Hoover, V. (2004). Research on competitive dynamics: Recent accomplishments and future challenges. Journal of Management, 30(6), 779-804. 

GRUPOS ESTRATÉGICOS EN EL SECTOR PRIVADO DE LA EDUCACIÓN SUPERIOR

Kwiek, M. (2017). De-privatization in higher education: a conceptual approach. Higher Education, 74(2), 259-281.

Kwiek, M. (2018). Private Higher Education in Developed Countries. In Shin J. \& P. Teixeira (Eds.), Encyclopedia of International Higher Education Systems and Institutions. https://doi.org/10.1007/978-94-0179553-1_92-1

Levy, D. (2009). Growth and Typology. In S. Bjarnason, K. Cheng, J. Fielden, M. Lemaitre, D. Levy, \& N. Varghese (Eds.), A New Dynamic: Private Higher Education (pp. 7-27), UNESCO.

Levy, D. (2013). The decline of private higher education. Higher Education Policy, 26(1), 25-42.

Meilich, O. (2019). Strategic groups maps: review, synthesis, and guidelines. Journal of Strategy and Management, 12(4), 447-463.

Navas, L. P., Montes, F., Abolghasem, S., Salas, R. J., Toloo, M., \& Zarama, R. (In press). Colombian higher education institutions evaluation. Socio-Economic Planning Sciences,

OECD. (2018). Education at a Glance 2018: OECD Indicators. http://dx.doi. org/10.1787/eag-2018-en

OECD (2016). La educación en Colombia: Revisión de politicas nacionales de eduación. http://dx.doi. org/10.1787/9789264250604-en
Raza, R. (2009). Examining Autonomy and Accountability in Public and Private Tertiary Institutions. Zhurnal Eksperimental'noi $i$ Teoreticheskoi Fiziki, 1-36.

Rossi, F. (2009). Increased competition and diversity in higher education: An empirical analysis of the Italian University system. Higher Education Policy, 22(4), 389-413.

Sagarra, M., Mar-Molinero, C., \& Rodríguez-Regordosa, H. (2015). Evaluating the success of educational policy in Mexican Higher Education. Higher Education, 69(3), 449-469.

Sánchez-Barrioluengo, M. (2014). Articulating the "three-missions" in Spanish universities. Research Policy, 43(10), 1760-1773.

Teixeira, P., Rocha, V., Biscaia, R., \& Cardoso, M. (2012). Competition and diversity in higher education: An empirical approach to specialization patterns of Portuguese institutions. Higher Education, 63(3), 337-352.

van Vught, F. A., Kaiser, F., File, J. M., Gaethgens, C., Peter, R., \& Westerheijden, D. (2010). U-Map. The European Classification of Higher Education Institutions, 1-46.

Wilkins, S. (2019). The positioning and competitive strategies of higher education institutions in the United Arab Emirates. International Journal of Educational Management, 34(1), 139-153. 
DORYS YANETH RODRIGUEZ CASTRO, JON MIKEL ZABALA-ITURRIAGAGOITIA, JUAN APARICIO

\section{PERFIL ACADÉMICO Y PROFESIONAL DE LOS AUTORES}

Dorys Yaneth Rodríguez Castro. ORCID: http://orcid.org/0000-00023824-0013

Doctoranda del Programa de Competitividad Empresarial y Territorial, Innovación y Sostenibilidad (CETIS) de la Deusto Business School, Universidad de Deusto en Donostia-San Sebastián (España). Su área de actuación es la gestión de la investigación y de la innovación en sistemas de políticas públicas, universidades y empresas. E-mail: djrc72@opendeusto.es

Jon Mikel Zabala-Iturriagagoitia. ORCID: http://orcid.org/0000-00031975-2555

Profesor e investigador en el Departamento de Economía de la Deusto Business School, Universidad de Deusto en Donostia-San Sebastián. Sus principales intereses docentes y de investigación se centran en las políticas de ciencia, tecnología e innovación y la gestión de la innovación. E-mail: jmzabala@deusto.es

Juan Aparicio. ORCID: http://orcid.org/0000-0002-0867-0004

Profesor Titular de Universidad en el Departamento de Estadística, Matemáticas e Informática y Director del Instituto Universitario de Investigación "Centro de Investigación Operativa” de la Universidad Miguel Hernández de Elche. Su línea principal de investigación es la evaluación de la eficiencia y la productividad desde un punto de vista metodológico y aplicado. E-mail: j.aparicio@umh.es

Fecha Recepción del Artículo: 05. Febrero. 2020

Fecha Modificación del Artículo: 11. Junio. 2020

Fecha Aceptación del Artículo: 11. Junio. 2020

Fecha Revisión para Publicación: 20. Junio. 2020 
